\title{
BMJ Open Examining the double burden of malnutrition for preschool children and women of reproductive age in low- income and middle-income countries: a scoping review protocol
}

\author{
Jason Mulimba Were (D) , ${ }^{1,2}$ Saverio Stranges, ${ }^{1,2,3,4}$ Ishor Sharma, ${ }^{1}$ \\ Juan Camilo Vargas-Gonzalez, ${ }^{1,5,6}$ M. Karen Campbell ${ }^{1,7,8,9}$
}

To cite: Were JM, Stranges S, Sharma I, et al. Examining the double burden of malnutrition for preschool children and women of reproductive age in low-income and middleincome countries: a scoping review protocol. BMJ Open 2021;11:e054673. doi:10.1136/ bmjopen-2021-054673

- Prepublication history for this paper is available online. To view these files, please visit the journal online (http://dx.doi. org/10.1136/bmjopen-2021 054673).

Received 19 June 2021 Accepted 19 November 2021

Check for updates

(c) Author(s) (or their employer(s)) 2021. Re-use permitted under CC BY-NC. No commercial re-use. See rights and permissions. Published by BMJ.

For numbered affiliations see end of article.

Correspondence to Jason Mulimba Were; jwere2@uwo.ca

\section{ABSTRACT}

Introduction The majority of the populations in lowincome and middle-income countries (LMICs) are encountering the double burden of malnutrition (DBM): the coexistence of both undernutrition and overnutrition sequalae. With DBM being a new phenomenon in research, little is known about its aetiology, operational definitions and risk factors influencing its manifestation. The proposed scoping review is aimed at mapping literature with regard to the DBM phenomenon among preschool children and women of reproductive age in LMICs who are among the most high-risk groups to encounter DBM.

Methods A comprehensive literature search will be conducted in the following electronic databases: MEDLINE, EMBASE, Scopus, CINAHL, LILACS and ProQuest Dissertations and Thesis Global. Additionally, searches in other government and institutional sources (WHO website and university repositories) and forward and backward citation tracking of seminal articles will also be done. Two reviewers will independently conduct title and abstract screening and full-text screening. Similarly, data extraction and coding will independently be done by two reviewers. Information extracted from included literature will be analysed qualitatively using thematic analysis approach and reported as per the Preferred Reporting Items for Systematic reviews and Meta-Analyses extension for Scoping Reviews guidelines.

Ethics and dissemination Ethical approval is not required for this study because the review is based on literature from publicly available sources. The dissemination of our findings will be done through presentations in relevant conferences and publication in a peer-reviewed journal.

\section{INTRODUCTION}

The modern-day world is characterised by the paradoxical co-occurrence of nutritionrelated diseases and conditions. On the one hand, overnutrition is fast becoming a global public health problem. Recent data from the WHO indicate that approximately 2 billion adults (above 18 years) and 40 million
Strengths and limitations of this study

To the best of our knowledge, this is the first scoping review that focuses on exploring the aetiology of the double burden of malnutrition among preschool children and women of reproductive age in low-income and middle-income countries.

- This review will encompass comprehensive literature search and will utilise a renowned thematic analysis framework to synthesis the findings of the study.

- The findings of this review will be important in both mapping the current literature with regard to the double burden of malnutrition phenomenon for high-risk populations and also guiding secondary data analysis for our subsequent studies.

- The anticipated dearth of causation literature and longitudinal studies in this area of research may limit our findings, specifically in understanding the aetiology of double burden of malnutrition.

preschool children (under 5 years of age) are overweight or obese. ${ }^{1}$ This is a likely reflection of the ongoing nutrition transition, primarily in low-income and middle-income countries (LMICs), that is, currently characterised by the progressive affinity for caloriedense foods and sedentary lifestyles in many population subgroups. ${ }^{23}$ On the other hand, nutritional deficiencies that are facilitated by food inadequacies still persists in many population subgroups around the world, namely in the Global South. ${ }^{2}$ Nearly half a billion adults have been estimated to be underweight, while 155 million preschool children have been reported to be stunted. ${ }^{1}$ This contemporaneous existence of multiple nutrition burdens has led to the emergence of the double burden of malnutrition (DBM), a construct that characterises the simultaneous presence 

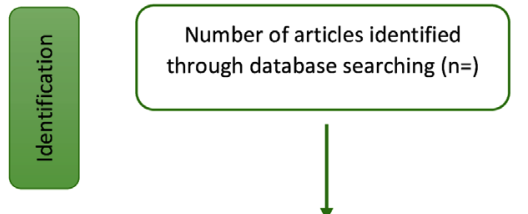

Additional records identified through other sources $(n=)$

Number of articles after removing the duplicates $(n=)$
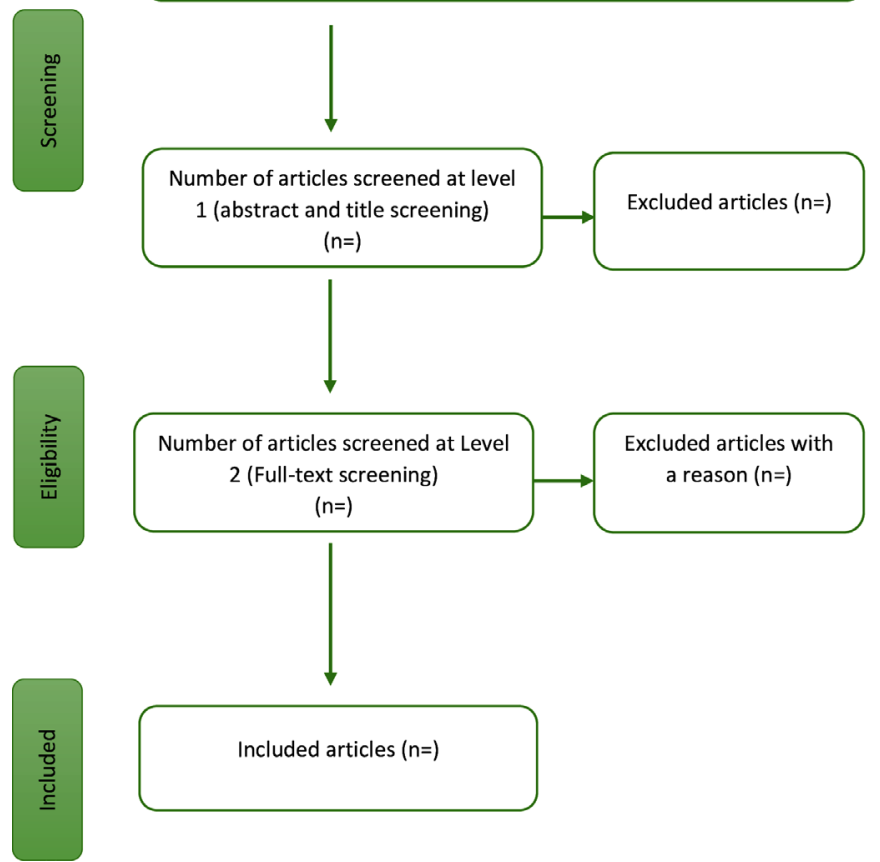

Included articles $(\mathrm{n}=)$

Figure 1 Scoping review flow diagram.

of both undernutrition and overnutrition sequalae. ${ }^{1}$ The DBM can be manifested at three different levels: ${ }^{1}$ individual level-depicting the co-occurrence of stunting, wasting or micronutrient deficiency with overweight/ obesity at a single point in time or across the life course (eg, stunting in childhood and being overweight and obese in adulthood as a result of irreversible metabolic programming in early life) $;{ }^{45}$ household level—where at least two household members have discordant nutritional statuses; ${ }^{1}$ and the population level—where both undernutrition and overnutrition types are common in the same population unit. ${ }^{1}$

Although everyone is susceptible to changes in nutritional health in response to sociodemographic changes, women of reproductive age (WRA) and preschool children are among the most vulnerable population groups. ${ }^{167}$ Recent estimates show that $9.27 \%$ of adult women globally are underweight, while $40.5 \%$ are overweight or obese. ${ }^{8}$ Conversely, $22.9 \%$ of children under the age of 5 are estimated to be stunted, whereas $6.0 \%$ are overweight or obese. ${ }^{9}$ These groups' risks of DBM are elevated due to the periods of growth and development that they are experiencing. Preschool children are at a critical window of rapid growth and development that requires optimal nutrition to support physical growth spurts experienced during this period. ${ }^{6}$ As for WRA, nutritional demand increases due the onset of menstruation, pregnancy and breastfeeding, hence their nutritional status is highly sensitive to deficiencies in diets. ${ }^{6}{ }^{10}$ Furthermore, some of the proximate risk factors of DBM among these two groups are by themselves influenced by broader sociodemographic factors acting at different levels of the society (eg, household, community and regional levels). ${ }^{6}$ For instance, increased urbanisation, industrialisation, economic development and global trade policy liberalisation have resulted to changes in dietary patterns and occupation structures, which in turn have had an influence on the individual's nutrition status. ${ }^{2710}$

The emergence of DBM creates an opportunity for health practitioners to identify population subgroups at most risk of adverse health outcomes. In recognition of this, the WHO in 2016 developed the double-duty actions, to provide a framework that leverages existing policies, interventions and programmes to concurrently reduce the risk of DBM. ${ }^{1}$ However, there is a dearth of studies from LMICs to spur the actualisation of this and other related frameworks. A major contributor to this scarcity is the recency of the concept of DBM in research. The definition of DBM as the coexistence of undernutrition and overnutrition within individual's, households, population or life-course is broad enough to capture the current nutrition realties in a lot of the world's population. ${ }^{611}$ However, this broad definition does not offer clear guidance on the specifics of measuring DBM. In extant literature, DBM has been defined in a myriad of ways, using varied nutritional biomarkers. ${ }^{611}$ For instance, anthropometric based measures such as Body Mass Index, waist circumference and waist-to-hip ratio for women and height-for-age and weight-for-height Z-scores for preschool children have commonly been in studies to operationally define DBM. ${ }^{11}$ Presumably, the thinking underlying these definitions have been based on study context, target population and data availability. ${ }^{11}$ Furthermore, there is no clear understanding as to whether the co-occurrence of the malnutrition indicators in the documented literature represents a non-random cluster with plausible similar aetiology. Therefore, the aim of this study is to map evidence of the DBM phenomenon in LMICs. It is anticipated that the proposed study will contribute to literature by adding knowledge on the plausible mechanisms for the existence of DBM at the individual, household and population levels among WRA and preschool children. Additionally, our findings are expected to guide future research on operational definitions and construct measurements that could be used in studying the DBM phenomenon among these vulnerable groups in LMICs.

\section{METHODS AND ANALYSIS}

A scoping review was determined as the most suitable literature synthesis design for this study given the exploratory nature of the study objective. ${ }^{13}$ After gaining familiarity with the current literature on DBM among the populations of interest, we established the following research 


\begin{tabular}{|c|c|}
\hline Category & Description \\
\hline Author/year & Author(s) name(s) and year of publication. \\
\hline Study title & Working title of the study. \\
\hline Aim/objectives & A description of the aim of the study as stated by the authors. \\
\hline Setting/context & $\begin{array}{l}\text { Country where the study was conducted. } \\
\text { Specific location in which the study was conducted (district/province/institution based). } \\
\text { Year the study was conducted. }\end{array}$ \\
\hline Methods & $\begin{array}{l}\text { Study design used to conduct the study (eg, cohort study, case-control, cross-sectional). } \\
\text { Participant's characteristics (specify salient characteristics of the target population, eg, sex and age bracket). } \\
\text { Data sources used in the study (primary or secondary-specify the source). } \\
\text { Sampling strategy: briefly specify how participants were identified and recruited the study. } \\
\text { Data collection: specify how data was gathered (eg, measurement tools, questionnaires, etc.). } \\
\text { Outcome measures: specify malnutrition indicators that have been used to measure nutritional status and how the } \\
\text { double burden of malnutrition was operationally defined. } \\
\text { Exposure measures: specify the main covariates that were studied. } \\
\text { Data analysis: specify the analytical strategy (eg, thematic analysis, logistic regression, etc.). }\end{array}$ \\
\hline Results & $\begin{array}{l}\text { Operational definition of DBM: describe all the findings with regards DBM phenotypes that have been used (ie, } \\
\text { nutritional biomarkers used in the definition and reported prevalence). } \\
\text { Explanations for the occurrence of DBM: extract any information that provides explanations/hypothesis for the co- } \\
\text { occurrence of the reported DBM phenotypes. } \\
\text { Exposure: discuss any reporting of factors (risk or protective factors) found to influence the occurrence of DBM. } \\
\text { Miscellaneous findings: report any other important findings. }\end{array}$ \\
\hline Limitations & Highlight the shortcomings of the study. \\
\hline
\end{tabular}

DBM, double burden of malnutrition.

objective. To describe the current state of knowledge regarding the DBM among preschool children and WRA in LMICs. This objective will be examined through the following research questions:

1. Which nutrition indicators have been used to operationally define the DBM at the individual, household and population levels among preschool children and WRA?

2. What are the posited explanations for the occurrence of the identified DBM phenotypes at the individual, household and population levels among preschool children and WRA?

3. What are the risk factors for the DBM at the individual, household and population levels among preschool children and WRA?

Although there are systematic reviews (both published and ongoing reviews) that have focused on the operational definitions of the DBM, ${ }^{11} 1{ }^{15}$ to the best of our knowledge, we found no existing reviews that succinctly synthesises literature on DBM phenomenon based on the review questions that we have formulated.

\section{Patient and public involvement}

There will be no patient and public involvement in this review.

\section{Eligibility criteria}

This study will use the Population-Concept-Context framework in determining the study's eligibility criteria. ${ }^{13}$
The populations of interest will be WRA (15-49 years) and children below the age of 5 years (0-59 months). The key concept is the DBM defined as the coexistence of undernutrition and overnutrition burdens. Studies will be included if they contain any of the following key concepts: measurement of DBM at the individual, household, population or life-course levels; theoretical frameworks, models or body of knowledge used to explain DBM phenotypes and risk factors for DBM. The context includes all studies that were conducted in LMICs-as defined by the world bank. ${ }^{16}$

There are no limitations with regard to the study design. Peer-reviewed articles, grey literature, theses, dissertations, published and unpublished reports will be included. Abstracts for which primary data or full-text literature can be accessed will also be included. All non-English studies will be included. In instances where studies are published in native languages, a native speaking person will be requested to translate the study into English. Furthermore, no limits will be placed with regard to the timeperiod of which the study was conducted. Studies that solely focus on men, children above the age of 5 years, adults above 50 years and LMIC migrants residing in highincome countries will be excluded. Furthermore, studies will be limited to those involving human participants.

\section{Search strategy}

Discussions with a library specialist and among coauthors identified relevant databases. A collective decision was 
to search the following electronic databases: MEDLINE, EMBASE, Scopus, CINAHL, LILACS and ProQuest Dissertations and Thesis Global. The search will be conducted using a combination of the subject headings and keywords relating to 'double burden of malnutrition', 'women of reproductive age', 'preschool children' and 'low- and middle-income countries'. Additionally, relevant grey literature from government sources and organisations such as the WHO will also be searched. After screening, forward and backward citation tracking of seminal studies will be done to identify all relevant articles missed in the database search. Authors of unpublished data will be contacted to provide any relevant information. After completing the searches, the reference list will be uploaded in EndNote citation management software $^{17}$ for deduplication and afterwards transferred to Rayyan software ${ }^{18}$ for screening purposes.

\section{Screening}

Screening will be done according to the Preferred Reporting Items for Systematic reviews and Meta-Analyses extension for Scoping Reviews (PRISMA-ScR) guidelines. ${ }^{13}$ Prior to the review screening, all reviewers will conduct a pilot screening of 10 randomly selected articles and discuss their findings to achieve consistency in the application of the eligibility criteria during the screening process. ${ }^{19}$ Afterwards, there will be two levels of article screening: level 1 will entail screening of study titles and abstract to eliminate obvious irrelevant articles. ${ }^{13}$ Level 2 will constitute full-text screening for all eligible studies against the inclusion/exclusion criteria. Both levels will be done by two reviewers independently. ${ }^{13}$ Disagreements will be resolved through discussions with a third reviewer. The PRISMA diagram will be used to highlight the outcome of the screening process (figure 1).

\section{Data charting process}

Two reviewers will independently extract data from the selected articles using a charting form developed by the review team. In general, the form will include bibliographic information, study characteristics (context, study design and participants characteristics), concept (malnutrition indicators, DBM definitions, level of DBM analysis, theoretical/conceptual frameworks), significant findings and conclusions (table 1). To ensure consistency and accuracy in recording data while using the charting form, a pilot test will be done by the reviewers independently on $10 \%$ of the included studies (randomly selected). ${ }^{19}$ The charting form will be calibrated based on the feedback from the pilot study. ${ }^{13}$ Afterwards, the review team will continuously update the charting form in an iterative manner to ensure all relevant information is captured during the charting process. Any disagreements will be resolved through discussion with a third reviewer.

\section{Data analysis and synthesis}

This study will present a qualitative synthesis of current literature performed using the inductive thematic analysis as suggested by Braun and Clarke. ${ }^{20}$ Extracted information will be subjected to content analysis, structured based on the review objectives as follows: study context, population type, malnutrition indicators, DBM phenotypes, explanations for the occurrence of DBM phenotypes and risk factors for DBM. Each reviewer will independently reread the extracted information and afterwards, generate codes and apply them to the excerpts of extracted information..$^{20}$ All the codes and related data excerpts will be collated and sorted into potential themes. ${ }^{20}$ The resulting themes will then be reviewed and refined by the review team with the aim of examining the relationship between the themes and the review questions..$^{20}$ Finally, a narrative synthesis and visual display (where possible) will be performed for the generated themes.

\section{DISCUSSION}

We believe that the information gathered in this review will provide an up-to-date understanding of DBM phenomenon in LMICs. Consequently, synthesis of DBM knowledge will provide a foundation for assessing the current operational definitions and identifying important knowledge gaps in literature. Furthermore, findings from the proposed scoping review will guide future analytical research including a secondary data analysis for the second phase of this study. It is anticipated that the proposed review will inform the modelling and interpretation of DBM outcomes for our subsequent studies. Additionally, the proposed review is expected to provide more insights on the potential risk factors of DBM and the inter-relationships among them which will be used to guide the development of statistical models (path diagrams) that would be empirically tested in the second phase of the study.

\section{Ethics and dissemination}

Ethical approval is not required for this study because the review is based on literature from publicly available sources. The dissemination of our findings will be done through conference presentations and publication in a peer-reviewed journal.

\section{Author affiliations}

${ }^{1}$ Epidemiology and Biostatistics, Schulich School of Medicine \& Dentistry, Western University, London, Ontario, Canada

${ }^{2}$ The Africa Institute, Western University, London, Ontario, Canada

${ }^{3}$ Department of Medicine, Schulich School of Medicine \& Dentistry, Western University, London, Ontario, Canada

${ }^{4}$ Department of Population Health, Luxembourg Institute of Health, Strassen, Luxembourg

${ }^{5}$ Instituto de Evaluación Tecnológica en Salud (IETS), Bogotá D.C, Colombia

${ }^{6}$ Unidad de Neurología, Hospital Universitario Nacional de Colombia, Bogotá D.C,

Colombia

${ }^{7}$ Department of Pediatrics, Schulich School of Medicine \& Dentistry, Western University, London, Ontario, Canada

${ }^{8}$ Department of Obstetrics \& Gynecology, Schulich School of Medicine \& Dentistry, Western University, London, Ontario, Canada

${ }^{9}$ Children's Health Research Institute, Lawson Health Research Institute, London, Ontario, Canada 
Twitter Jason Mulimba Were @jasonmulimba

Acknowledgements We would like to express our gratitude to Piotr Wilk and Shehzad Ali (Faculty members, Department of Epidemiology and Biostatistics at Western University) for their advisory support for this project. We would also like to thank Marisa Tippet (Research and Scholarly Communications librarian at Western university) for her technical guidance in the development of the study's methods section.

Contributors JMW, SS and MKC decided on the area of research. JMW, SS, IS, JCV-G and MKC conceptualised the research, contributed to the development of the review questions and writing of the manuscript. SS and MKC were responsible for supervising the project. JMW was responsible for the final editing, formatting and submission.

Funding The authors have not declared a specific grant for this research from any funding agency in the public, commercial or not-for-profit sectors.

Competing interests None declared.

Patient and public involvement Patients and/or the public were not involved in the design, or conduct, or reporting or dissemination plans of this research.

Patient consent for publication Not required.

Provenance and peer review Not commissioned; externally peer reviewed.

Data availability statement Data sharing not applicable as no data sets generated and/or analysed for this study. All the information used in this scoping review protocol are from publicly available sources.

Open access This is an open access article distributed in accordance with the Creative Commons Attribution Non Commercial (CC BY-NC 4.0) license, which permits others to distribute, remix, adapt, build upon this work non-commercially, and license their derivative works on different terms, provided the original work is properly cited, appropriate credit is given, any changes made indicated, and the use is non-commercial. See: http://creativecommons.org/licenses/by-nc/4.0/.

\section{ORCID iD}

Jason Mulimba Were http://orcid.org/0000-0003-4288-7327

\section{REFERENCES}

1 World Health Organization. Double-duty actions for nutrition: Policy Brief [Internet], 2017. Available: https://www.who.int/publications/i/ item/WHO-NMH-NHD-17.2

2 Abrahams Z, Mchiza Z, Steyn NP. Diet and mortality rates in subSaharan Africa: stages in the nutrition transition. BMC Public Health 2011;11:1-12.

3 Popkin BM. The shift in stages of the nutrition transition in the developing world differs from past experiences! Malays J Nutr 2002;8:109-24.
4 Sawaya AL, Roberts S. Stunting and future risk of obesity: principal physiological mechanisms. Cad Saude Publica 2003;19 Suppl 1:S21-8.

5 Fall CHD, Kumaran K. Metabolic programming in early life in humans. Phil. Trans. R. Soc. B 2019;374:20180123.

6 Wells JC, Sawaya AL, Wibaek R, et al. The double burden of malnutrition: aetiological pathways and consequences for health Lancet 2020;395:75-88. doi:10.1016/S0140-6736(19)32472-9

7 Were JM, Stranges S, Creed IF. Fertility is a key predictor of the double burden of malnutrition among women of child-bearing age in sub-Saharan Africa. J Glob Health 2020;10:1-11.

8 NCD Risk Factor Collaboration (NCD-RisC). Trends in adult bodymass index in 200 countries from 1975 to 2014: a pooled analysis of 1698 population-based measurement studies with $19 \cdot 2$ million participants. Lancet 2016;387:1377-96. doi:10.1016/S01406736(16)30054-X

9 UNICEF/WHO/World Bank Group. Levels and trends in child malnutrition, 2017. Available: https://www.who.int/nutgrowthdb/jme_ brochoure2017.pdf

10 Popkin BM, Corvalan C, Grummer-Strawn LM. Dynamics of the double burden of malnutrition and the changing nutrition reality. Lancet 2020;395:65-74. doi:10.1016/S0140-6736(19)32497-3

11 Davis J, Oaks B, Engle-Stone R. The double burden of malnutrition: a systematic review of operational definitions (P22-006-19). Curr Dev Nutr 2019:3:1-14.

12 Engle-Stone R, Guo J, Ismaily S, et al. Intraindividual double burden of overweight and micronutrient deficiencies or anemia among preschool children. Am J Clin Nutr 2019;112:478S-87.

13 Tricco AC, Lillie E, Zarin W, et al. PRISMA extension for scoping reviews (PRISMA-ScR): checklist and explanation. Ann Intern Med 2018;169:467-73.

14 Tan X, Tan PY. The evidence for the association between micronutrient deficiencies and over- or undernutrition: a systematic review, 2020. Available: https://www.crd.york.ac.uk/prospero/ display_record.php?ID=CRD42020152975

15 Kosaka S, Umezaki M. A systematic review of the prevalence and predictors of the double burden of malnutrition within households. $\mathrm{Br}$ J Nutr 2017;117:1118-27.

16 Prydz EB, Wadhwa D. Classifying countries by income, 2019. The world bank. Available: https://datatopics.worldbank.org/worlddevelopment-indicators/stories/the-classification-of-countries-byincome.html\#: :text=As of 1 July 2019, between \%243\%2C996 and $\% 2412 \% 2$ C375\%3B high- [Accessed 31 May 2021].

17 The EndNote Team. Endnote. Philadelphia: Clarivate, 2013.

18 Ouzzani M, Hammady H, Fedorowicz Z, et al. Rayyan-a web and mobile APP for systematic reviews. Syst Rev 2016;5:210. doi:10.1186/s13643-016-0384-4

19 Julian PTH, Thomas J, Chandler J. Selecting studies and collecting data. In: Cochrane handbook for systematic reviews of interventions, 2008: 151-83. https://training.cochrane.org/handbook

20 Braun V, Clarke V. Using thematic analysis in psychology. Qual Res Psychol 2006;3:77-101. 